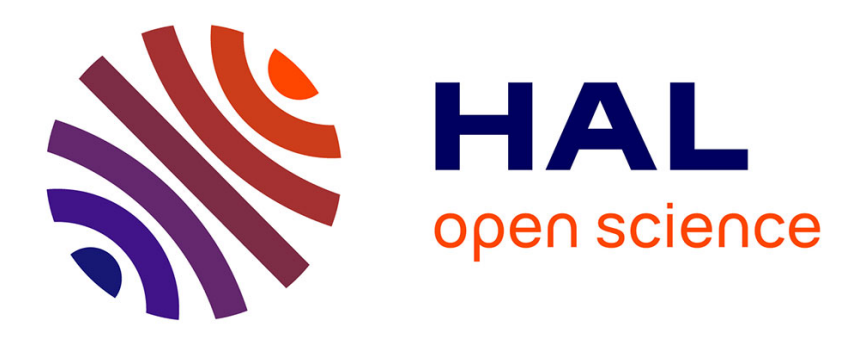

\title{
Moments of first passage times in general birth-death processes
}

Oualid Jouini, Yves Dallery

\section{To cite this version:}

Oualid Jouini, Yves Dallery. Moments of first passage times in general birth-death processes. Mathematical Methods of Operations Research, 2008, 68, pp.49-76. 10.1007/s00186-007-0174-9 . hal01264961

\section{HAL Id: hal-01264961 \\ https://hal.science/hal-01264961}

Submitted on 3 Feb 2016

HAL is a multi-disciplinary open access archive for the deposit and dissemination of scientific research documents, whether they are published or not. The documents may come from teaching and research institutions in France or abroad, or from public or private research centers.
L'archive ouverte pluridisciplinaire HAL, est destinée au dépôt et à la diffusion de documents scientifiques de niveau recherche, publiés ou non, émanant des établissements d'enseignement et de recherche français ou étrangers, des laboratoires publics ou privés. 


\title{
Moments of First Passage Times in General Birth-Death Processes
}

\author{
Oualid Jouini \& Yves Dallery \\ Laboratoire Génie Industriel, Ecole Centrale Paris \\ Grande Voie des Vignes, 92295 Châtenay-Malabry Cedex, France. \\ walid.jouini@ecp.fr, yves.dallery@ecp.fr
}

To appear in Mathematical Methods of Operations Research, May 2007.

\begin{abstract}
We consider ordinary and conditional first passage times in a general birth-death process. Under existence conditions, we derive closed-form expressions for the $k$ th order moment of the defined random variables, $k \geq 1$. We also give an explicit condition for a birth-death process to be ergodic degree 3. Based on the obtained results, we analyze some applications for Markovian queueing systems. In particular, we compute for some non-standard Markovian queues, the moments of the busy period duration, the busy cycle duration, and the state-dependent waiting time in queue.
\end{abstract}

keywords Birth-Death Processes; First Passage Times; Conditional First Passage Times; Busy Period; Transient Analysis

Short title: Analysis of Birth-Death Processes

Corresponding author: Oualid Jouini (walid.jouini@ecp.fr)

Tel: +33141131166 / Fax: +33141131272

\section{Introduction}

The literature dealing with the study of queueing systems is huge. The analytical studies were intended to obtain useful information for the decision making process, basically related to the design, the control, and the measurement of effectiveness of the systems. Birth-death processes, and in general Markov chains, are broadly used in the field of queueing theory. They are a rich and important class in modeling numerous phenomena in queueing systems. In this paper, we are dealing with the transient (time-dependent) analysis of birth-death processes. The characteristics of interest are the ordinary and conditional first passage times. These characteristics are known to be of value for the performance evaluation of several queueing systems. Analyzing either transient or stationary queueing delays and response times, for instance, may be addressed by means of ordinary and conditional first passage times.

For the time-dependent solutions, advanced mathematical techniques are necessary. The well studied systems are the simple ones, namely, $M / M / 1 / K, M / M / 1$, and $M / M / \infty$, see Kleinrock [23], and Gross and Harris [11]. Such solutions are due first to Bailey [5]'s work where the author has solved the partial differential equations governing the underlying birth-death process via generating functions. Another interesting approach, based on advanced combinatorial methods, was done by Champernowne [8]. In general, most of the popular procedures derive the transient expressions using a combination of generating probability functions and Laplace transforms, see Abate and 
Whitt [1], Parthasarathy [29], and Abate and Whitt [2] for an overview. In these papers, the numerical solutions are complex due to the use of Bessel functions. Some other approaches were proposed as a method based on Taylor series in Krinik and Sourouri [25], and a new method based on the uniformization technique and on generating functions proposed by Leguesdron et al. [26]. The last method is of interest in the sense that it leads to quite simple expressions for the transient probabilities. For the $M / M / 1 / K$, Tarabia [34] gave an alternative simple approach to the procedure of Takâcs [33]. He showed that the measures of effectiveness such as the first and the second order moments of the queue length can be easily obtained in a new and elegant closed-form. The result was also derived for the $M / M / 1$ case by taking the limit as $K \rightarrow \infty$.

The literature specifically related to birth-death processes is extensive and growing, see Karlin and McGregor [16, 17], Keilson [20, 21], Sumita [32], Mao [28], Guillemin [12], and Coolen-Schrijner and van Doorn [9]. We refer the reader to Keilson [18, 19], and Kijima [22] for an overview on the subject. In Guillemin and Pinchon [13], the authors revisited the resolution of the forward Chapman-Kolmogorov equations associated with a birth-death process through the spectral theory. Their work was based on the connection between probability theory and continued fractions addressed first by Karlin and McGregor [17]. They investigated, specifically, how Laplace Transforms of different transient characteristics related to excursions in a general birth-death process can be expressed by means of the basic orthogonal polynomials system and the spectral measure. Flajolet and Guillemin [10] have developed a formal calculus of basic events described by lattice paths associated with birth-death processes. They expressed several basic events in terms of continued fractions and their associated orthogonal polynomials. An extension of the latter paper was developed in Ball and Stefanov [6], where the authors have used an approach based on viewing birth-death processes as exponential families.

In this paper, we consider the transient behavior of general birth-death processes. We mean by "general" that transition rates are arbitrary and need not have some special structure. Using the associated Chapman-Kolmogorov equations, and via Laplace transforms we derive closed-form expressions for the moments of ordinary and conditional downcrossing and upcrossing times between pairs of states. Further interesting characteristics are sojourn times in states. This topic is out of the scope of this paper. As we shall mention in the paper, some of our results about the ordinary downcrossing and upcrossing times were derived in the literature using a different approach. The existing results lead to a representation of Laplace transforms of transient characteristics in terms of continued fractions and orthogonal polynomials. Although continued fractions are known to be useful especially for numerical issues, few of their closed-form expressions are available. The known expressions deal with simple models such as the $M / M / 1$ and $M / M / \infty$ queues. The analysis in this paper allows us however to address several further applications. We show the equivalence between the analysis of various characteristics in some examples of queueing systems and that of hitting and return times. Also, we recover in a simple way some classical results such as the busy period and busy cycle durations in basic Markovian systems.

The rest of the paper is organized as follows. In Section 2, we specify the general birthdeath process we consider. In Sections 2.1 and 2.2, we define two families of random variables; the 
unconditional (ordinary) first passage times and the conditional first passage times, respectively. In Section 3, we derive the moments for the ordinary random variables. In Section 4, we apply the same analysis for the conditional random variables. In Section 5 , we investigate various applications of the analysis of the ordinary first passage times. In Section 6, we close the paper by some concluding remarks and possible future research.

\section{Model Description and Notations}

We consider a continuous-time birth-death process $\{E(t), t \geq 0\}$ with discrete state space taking non-negative integer values $\{0,1,2,3, \ldots\}$ defined on a probability space. The transition rates of the process $\{E(t), t \geq 0\}$ are denoted by

$$
q_{m, m+1}=\lambda_{m}>0, q_{m, m-1}=\mu_{m}, q_{m, m}=-\left(\lambda_{m}+\mu_{m}\right) \text { for } m \geq 0 \text {, and } q_{m, n}=0 \text { otherwise. }
$$

The rate $\mu_{0}$ is equal to 0 , and $\mu_{m}>0$ for $m>0$. For $m \geq 0$, we define the quantities $\pi_{m}$ by

$$
\pi_{0}=1, \text { and } \pi_{m}=\frac{\lambda_{0} \ldots \lambda_{m-1}}{\mu_{1} \ldots \mu_{m}} \text { for } m \geq 1 .
$$

The quantities $\pi_{m}$ are called the potential coefficients of the birth-death process $\{E(t), t \geq 0\}$. Starting from a given initial state, let the transient probabilities be $\left\{p_{m}(t), t \geq 0\right\}, m \geq 0$. The quantity $p_{m}(t)$ represents the probability that at an arbitrary time $t$, the system is in state $m$, $m \geq 0$. Under the condition of existence, the stationary distribution of the process $\{E(t), t \geq 0\}$ defined for $m \geq 0$ by the quantities $p_{m}=\lim _{t \rightarrow \infty} p_{m}(t)$ can be easily solved through recursion. They are given by

$$
p_{m}=\frac{\pi_{m}}{\sum_{i=0}^{\infty} \pi_{i}}>0, \text { for } m \geq 0
$$

Further details about the condition of existence of these quantities will be given in Section 3 .

\subsection{First Passage Times}

In this section, we define the random variables associated with first passage times in birth-death processes. Let us consider the random variable $\theta_{m}$ representing the duration of an excursion by the process $\{E(t), t \geq 0\}$ above the level $m-1, m \geq 1$. In other words, $\theta_{m}$ represents the first passage time from state $m$ to state $m-1$. We define $\theta_{m}$ by

$$
\theta_{m}=\operatorname{Inf}\{t>0: E(t)=m-1 \mid E(0)=m\}
$$

Also, let $\tau_{m}$ be the first passage time from state $m-1$ to state $m$, defined by

$$
\tau_{m}=\operatorname{Inf}\{t>0: E(t)=m \mid E(0)=m-1\} .
$$

Let us now introduce the random variables $D_{m}$ and $U_{m}$ representing the downcrossing time from state $m$ to the empty state 0 , and the upcrossing time from state 0 to state $m$, respectively. 
These random variables are given by

$$
\begin{gathered}
D_{m}=\operatorname{Inf}\{t>0: E(t)=0 \mid E(0)=m\}, \\
\text { and, } U_{m}=\operatorname{Inf}\{t>0: E(t)=m \mid E(0)=0\} .
\end{gathered}
$$

One can easily see, for $m \geq 1$, that

$$
\begin{gathered}
D_{m} \doteq \sum_{n=1}^{m} \theta_{n}, \\
\text { and, } U_{m} \doteq \sum_{n=1}^{m} \tau_{n},
\end{gathered}
$$

where $\doteq$ denotes equality in terms of distributions. In general concerning first passage times between two different states, one may define the random variables of the downcrossing or upcrossing times from any state $i$ to any state $j, i \neq j$. We notice that the analysis we provide in this paper will easily cover these quantities.

Finally, let $C_{m}$ be the random variable denoting the time between two visits by the process $E(t)$ at state 0 , giving that the process $E(t)$ hits state $m$. Then, we state that

$$
C_{m} \doteq U_{m}+D_{m} \doteq \sum_{n=1}^{m}\left(\theta_{n}+\tau_{n}\right)
$$

The analysis of the random variables defined above is useful for various problems in Markovian queueing systems. For instance, it would be helpful for the busy period analysis of queueing systems with state-dependent arrival and service rates. We can also use it for computing the characteristics of the state-dependent waiting time distribution in complex service systems. We shall give further details of these applications in Section 5.

\subsection{Conditional First Passage Times}

The random variables of first passage times, we defined above, have great importance in Markovian queueing systems applications. Of equally great interest are the conditional first passage times. In what follows, we define their associated random variables.

Let ${ }^{r} \theta_{m}$ be the first passage time of the process $\{E(t), t \geq 0\}$ from state $m$ to state $m-1$ given that the process does not visit state $r$ in between, $1 \leq m<r$, defined by

$$
{ }^{r} \theta_{m}=\operatorname{Inf}\{t>0: E(t)=m-1 \mid E(0)=m \text { and no visit to } r\} .
$$

Similarly, let ${ }^{r} \tau_{m}$ be the first passage time from state $m-1$ to state $m$ given no visit to $r$, $0 \leq r<m-1$, defined by

$$
{ }^{r} \tau_{m}=\operatorname{Inf}\{t>0: E(t)=m \mid E(0)=m-1 \text { and no visit to } r\} .
$$

As above, one may also define the conditional downcrossing and upcrossing times between two different states, given no visit to a third state. Using the results we give in Section 4, the analysis 
of these quantities can be easily done.

In practice, we find several useful applications for the conditional first passage times. One interesting application would be for a make-to-stock system. Consider an inventory system with finite capacity ( $K_{1}$ items at most) in which demands are backlogged if no items are available for them upon arrival. There is a single machine with a finite queue size. A maximum of $K_{2}$ customers can be accepted in queue. A customer who finds a full waiting line is lost. In case of Markovian interarrival demand and production processing times, this system can be modeled as a birth-death process. In practice, it is useful to determine the time from an idle system (with no items in stock and no waiting customers) until a full inventory (with $K_{1}$ items in stock) given no backlogged demands in between. This is equivalent to compute the conditional first passage time of a particle (in the associated birth-death process) from the "idle" state up to the "full" inventory state, given that it does not visit the state with one backlogged customer. The latter state is the one just before the "idle" state. Another interesting performance measure is to compute the time from a full waiting line ( $K_{2}$ waiting customers) until all customers are served given no lost customers. This is again equivalent to compute a conditional first passage time in the associated birth death process. We notice in addition that evaluating these performances would allow to optimize the system parameters such as the inventory capacity, $K_{1}$, and the waiting line size, $K_{2}$.

\section{Moments of First Passage Times}

In this section, we focus on calculating the $k$ th order moment, $k \geq 1$, for the ordinary first passage times defined in Section 2.1. Before moving on to the moments computation, we should first discuss their conditions of existence. For the upcrossing times, $\tau_{m}$ and $U_{m}$, it is clear that no specific conditions are required. However, this is not the case for the downcrossing times, $\theta_{m}, D_{m}$, and neither for $C_{m}$. To guarantee the existence of the moments of these return times, we shall use the following set of conditions.

\section{Condition $C^{k}(k \geq 1)$ : the birth-death process $\{E(t), t \geq 0\}$ has ergodic degree $k$.}

Roughly speaking, the ergodic degree gives the number of finite moments possessed by the time of the first passage at a given state $i$ starting from any state $j \neq i$. We refer the reader to Mao [28] for more details. In particular, Condition $C^{1}$ simply reflects the classical ergodicity assumption: the condition under which the process settles into equilibrium (the birth rates are not too large relative to the death rates). This is equivalent to say that Condition $C^{1}$ is the necessary and sufficient condition for the expectation of the first passage time from any state $i$ to any state $j \neq i$ to be finite. From Karlin and McGregor [16], Condition $C^{1}$ holds if and only if

$$
\sum_{m=0}^{\infty} \pi_{m}<\infty \text { and } \sum_{m=0}^{\infty} \frac{1}{\lambda_{m} \pi_{m}}=\infty
$$

see also Kleinrock [23] and Keilson [19]. We notice from Kleinrock [23] that Condition $C^{1}$ is met whenever the sequence $\left\{\lambda_{m} / \mu_{m}\right\}$ remains below unity from some $m$ onwards, i.e., if there exists some $m_{0}$ such that for all $m \geq m_{0}$ we have $\lambda_{m} / \mu_{m}<1$. We will find this to be true in the applications we consider in Section 5 . 
From Theorem 6.1 in Coolen-Schrijner and van Doorn [9], Condition $C^{2}$ holds if and only if

$$
\left(\sum_{j=0}^{\infty} \pi_{j}\right)^{-1} \cdot\left(\sum_{m=0}^{\infty} \frac{1}{\lambda_{m} \pi_{m}}\left(\sum_{j=m+1}^{\infty} \pi_{j}\right)^{2}\right)<\infty
$$

An equivalent expression was also found in Theorem 4 of Karlin and McGregor [16].

We should note that if a birth-death process has ergodic degree $k$, then it has also all ergodic degrees $l$ with $1 \leq l \leq k$. An explanation would be as follows. Let us consider a birth-death process which has ergodic degree $k, k \geq 1$. Then, the $k$ th order moment of the first passage time from any state $i$ to any state $j \neq i$ is finite. From Loève [27], we state that if the $k$ th order moment of a given positive random variable is finite, hence, any $l$ th order moment of that random variable is also finite, $1 \leq l \leq k$. As a consequence for our birth-death process, any $l$ th order moment of the first passage time from any state $i$ to any state $j \neq i$ is finite, $1 \leq l \leq k$. Finally, our birth-death process has ergodic degree $l$ with $1 \leq l \leq k$.

For instance, one may check that Condition $C^{2}$ guarantees Condition $C^{1}$. Assume therefore that Relation (14) holds, and let us prove that Relation (13) holds too. Having $\pi_{0}=1$, the left hand side of Relation (14) may be rewritten as

$$
\begin{aligned}
\left(\sum_{j=0}^{\infty} \pi_{j}\right)^{-1} & \cdot\left(\sum_{m=0}^{\infty} \frac{1}{\lambda_{m} \pi_{m}}\left(\sum_{j=m+1}^{\infty} \pi_{j}\right)^{2}\right)= \\
& \left(1+\sum_{j=1}^{\infty} \pi_{j}\right)^{-1} \cdot\left(\frac{1}{\lambda_{0}}\left(\sum_{j=1}^{\infty} \pi_{j}\right)^{2}+\sum_{m=1}^{\infty} \frac{1}{\lambda_{m} \pi_{m}}\left(\sum_{j=m+1}^{\infty} \pi_{j}\right)^{2}\right) .
\end{aligned}
$$

Knowing that all parameters are strictly positive reals, Equation (15) becomes

$$
\left(\sum_{j=0}^{\infty} \pi_{j}\right)^{-1} \cdot\left(\sum_{m=0}^{\infty} \frac{1}{\lambda_{m} \pi_{m}}\left(\sum_{j=m+1}^{\infty} \pi_{j}\right)^{2}\right)=\frac{1}{\lambda_{0}}\left(1+\sum_{j=1}^{\infty} \pi_{j}\right)^{-1} \cdot\left(\sum_{j=1}^{\infty} \pi_{j}\right)^{2}+K,
$$

where $K$ is a given strictly positive real. Then, Relation (14) leads to

$$
\frac{1}{\lambda_{0}}\left(1+\sum_{j=1}^{\infty} \pi_{j}\right)^{-1} \cdot\left(\sum_{j=1}^{\infty} \pi_{j}\right)^{2}<\infty
$$

Assume that $\sum_{j=0}^{\infty} \pi_{j}=\infty$. So, $\sum_{j=1}^{\infty} \pi_{j}=\infty$, which implies that Relation (17) is false. Hence, $\sum_{j=1}^{\infty} \pi_{j}<\infty$, and equivalently, $\sum_{j=0}^{\infty} \pi_{j}<\infty$. Let us now prove that $\sum_{j=0}^{\infty} \frac{1}{\lambda_{j} \pi_{j}}=\infty$. From Theorem 15 in Karlin and McGregor [17], we state that our birth-death process has one and only one transition probability matrix if and only if $\sum_{j=0}^{\infty}\left(\pi_{j}+\frac{1}{\lambda_{j} \pi_{j}}\right)=\infty$. Since $\sum_{j=0}^{\infty} \pi_{j}<\infty$, the series $\sum_{j=0}^{\infty} \frac{1}{\lambda_{j} \pi_{j}}$ necessarily diverges. Finally, Condition $C^{1}$ holds.

To the best of our knowledge, no explicit expressions for, $k \geq 3$, exist in the literature. We will give further details about Conditions $C^{k}$ with higher orders at the end of this section. In addition, we derive a new result by giving Condition $C^{3}$ in an explicit form. 
In Theorem 1, we give the $k$ th order moment expression of the random variable $\theta_{m}$. Thereafter, we deduce the expectation and the variance of $\theta_{m}$ in Corollaries 1 and 2 , respectively. In Theorem 2 , Corollaries 3 and 4 , a similar analysis is given for the random variable $\tau_{m}$. We Notice that the expressions of $E\left(\theta_{m}^{k}\right)$, for $k \geq 3$, and $E\left(\tau_{m}^{k}\right)$, for $k \geq 2$, are new.

For the rest of the paper, an empty sum is being interpreted as zero, and an empty product is being interpreted as one.

Theorem 1 Under Condition $C^{k}$, the $k$ th order moment $E\left(\theta_{m}^{k}\right), k \geq 1$, of the random variable $\theta_{m}, m \geq 1$, is given by

$$
E\left(\theta_{m}^{k}\right)=\frac{1}{\lambda_{m-1} \pi_{m-1}} \sum_{n=m}^{\infty} \lambda_{n-1} \pi_{n-1} V_{n, k},
$$

where

$$
V_{n, k}=\frac{k}{\mu_{n}} E\left(\theta_{n}^{k-1}\right)+\frac{\lambda_{n}}{\mu_{n}} \sum_{j=1}^{k-1}\left(\begin{array}{c}
k \\
j
\end{array}\right) E\left(\theta_{n}^{j}\right) E\left(\theta_{n+1}^{k-j}\right), \text { for } n \geq 1, k \geq 1 .
$$

Proof. From the Strong Markov Property, we can write, for $m \geq 1$

$$
\left\{\begin{array}{lll}
\theta_{m} \doteq \varepsilon_{\lambda_{m}+\mu_{m}} & , \text { with probability } \frac{\mu_{m}}{\lambda_{m}+\mu_{m}} \\
\theta_{m} \doteq \varepsilon_{\lambda_{m}+\mu_{m}}+\theta_{m+1}+\hat{\theta}_{m} & , \text { with probability } \frac{\lambda_{m}}{\lambda_{m}+\mu_{m}}
\end{array}\right.
$$

where $\varepsilon_{\lambda_{m}+\mu_{m}}$ is a random variable exponentially distributed with parameter $\lambda_{m}+\mu_{m}$. The random variables $\theta_{m}, \theta_{m+1}$ and $\hat{\theta}_{m}$ are independent. In addition, the random variables $\theta_{m}$ and $\hat{\theta}_{m}$ are identically distributed.

Let $\tilde{\theta}_{m}(s)$ be the Laplace transform of the random variable $\theta_{m}$. Then, Equation (20) yields

$$
\left(\lambda_{m}+\mu_{m}+s\right) \tilde{\theta}_{m}(s)=\mu_{m}+\lambda_{m} \tilde{\theta}_{m+1}(s) \tilde{\theta}_{m}(s), \text { for } m \geq 1
$$

Let $\tilde{\theta}_{m}^{(k)}(s)$ be the $k$ th derivative in $s$ of $\tilde{\theta}_{m}(s)$. Taking the $k$ th derivative in $s$ of both sides in Equation (21) using Leibnitz's differentiation formula, we obtain for $m \geq 1, k \geq 1$

$$
\left(\lambda_{m}+\mu_{m}+s\right) \tilde{\theta}_{m}^{(k)}(s)+k \tilde{\theta}_{m}^{(k-1)}(s)=\lambda_{m} \sum_{j=0}^{k}\left(\begin{array}{l}
k \\
j
\end{array}\right) \tilde{\theta}_{m}^{(j)}(s) \tilde{\theta}_{m+1}^{(k-j)}(s)
$$

or equivalently

$$
\begin{aligned}
\left(\lambda_{m}+\mu_{m}+s\right) \tilde{\theta}_{m}^{(k)}(s)+k \tilde{\theta}_{m}^{(k-1)}(s)= & \lambda_{m} \tilde{\theta}_{m}(s) \tilde{\theta}_{m+1}^{(k)}(s)+\lambda_{m} \sum_{j=1}^{k-1}\left(\begin{array}{c}
k \\
j
\end{array}\right) \tilde{\theta}_{m}^{(j)}(s) \tilde{\theta}_{m+1}^{(k-j)}(s) \\
& +\lambda_{m} \tilde{\theta}_{m}^{(k)}(s) \tilde{\theta}_{m+1}(s) .
\end{aligned}
$$

For $m \geq 1$ and $j=0, \tilde{\theta}_{m}^{(j)}(0)=1$. For $m \geq 1$ and $j \geq 1, \tilde{\theta}_{m}^{(j)}(0)=(-1)^{j} E\left(\theta_{m}^{j}\right)$. Hence, Equation 
(23) becomes for $s=0$

$$
\begin{gathered}
\left(\lambda_{m}+\mu_{m}\right)(-1)^{k} E\left(\theta_{m}^{k}\right)+k(-1)^{k-1} E\left(\theta_{m}^{k-1}\right)=\lambda_{m}(-1)^{k} E\left(\theta_{m+1}^{k}\right)+\lambda_{m}(-1)^{k} E\left(\theta_{m}^{k}\right) \\
+\lambda_{m} \sum_{j=1}^{k-1}\left(\begin{array}{c}
k \\
j
\end{array}\right)(-1)^{k} E\left(\theta_{m}^{j}\right) E\left(\theta_{m+1}^{k-j}\right) .
\end{gathered}
$$

Dividing by $(-1)^{k} \mu_{m}$ implies

$$
E\left(\theta_{m}^{k}\right)=\frac{\lambda_{m}}{\mu_{m}} E\left(\theta_{m+1}^{k}\right)+\frac{k}{\mu_{m}} E\left(\theta_{m}^{k-1}\right)+\frac{\lambda_{m}}{\mu_{m}} \sum_{j=1}^{k-1}\left(\begin{array}{c}
k \\
j
\end{array}\right) E\left(\theta_{m}^{j}\right) E\left(\theta_{m+1}^{k-j}\right)
$$

which leads to the following recurrence relation

$$
E\left(\theta_{m}^{k}\right)=\frac{\lambda_{m}}{\mu_{m}} E\left(\theta_{m+1}^{k}\right)+V_{m, k}, \quad m \geq 1, \quad k \geq 1 .
$$

With straightforward manipulations in Equation (26), we state that for $m \geq 1, i \geq 1$, and $k \geq 1$

$$
E\left(\theta_{m}^{k}\right)=V_{m, k}+\frac{\lambda_{m}}{\mu_{m}} V_{m+1, k}+\ldots+\left(\prod_{j=0}^{i-1} \frac{\lambda_{m+j}}{\mu_{m+j}}\right) V_{m+i, k}+\left(\prod_{j=0}^{i} \frac{\lambda_{m+j}}{\mu_{m+j}}\right) E\left(\theta_{m+i+1}^{k}\right) .
$$

For a given $k \geq 1$ and under the Condition $C^{k}$, we deduce that $E\left(\theta_{m}^{k}\right)$ is bounded for $m \geq 1$. Moreover, the birth-death process has, in particular, ergodic degree 1 . Then $\lim _{i \rightarrow \infty} \pi_{i}=0$, also, $\lim _{i \rightarrow \infty} \prod_{j=0}^{i} \frac{\lambda_{m+j}}{\mu_{m+j}}=0$, for $m \geq 1$. Hence, it follows that $\lim _{i \rightarrow \infty}\left(\prod_{j=0}^{i} \frac{\lambda_{m+j}}{\mu_{m+j}}\right) E\left(\theta_{m+i+1}^{k}\right)=0$, for $m \geq 1, k \geq 1$.

Continuing forward manipulations in Equation (27) until $i$ goes to $\infty$ implies

$$
E\left(\theta_{m}^{k}\right)=\sum_{i=0}^{\infty}\left(\prod_{j=0}^{i-1} \frac{\lambda_{m+j}}{\mu_{m+j}}\right) V_{m+i, k}, m \geq 1, k \geq 1
$$

Finally, observing that $\frac{1}{\lambda_{n}} \prod_{j=m}^{n} \frac{\lambda_{j}}{\mu_{j}}=\frac{\pi_{n}}{\lambda_{m-1} \pi_{m-1}}$, for $n \geq m \geq 1$, and through a change in the subscripts we obtain

$$
E\left(\theta_{m}^{k}\right)=\frac{1}{\lambda_{m-1} \pi_{m-1}} \sum_{n=m}^{\infty} \lambda_{n-1} \pi_{n-1} V_{n, k}, m \geq 1, k \geq 1,
$$

which completes the proof.

Corollary 1 Under Condition $C^{1}$, the expectation $\bar{\theta}_{m}$ of the random variable $\theta_{m}$ is given by

$$
\bar{\theta}_{m}=\frac{1}{\lambda_{m-1} \pi_{m-1}} \sum_{n=m}^{\infty} \pi_{n}, \text { for } m \geq 1
$$

Proof. For $n \geq 1$ and $k=1$, we have $V_{n, k}=\frac{1}{\mu_{n}}$. Then, applying Theorem 1 easily leads to the desired result. 
The result in Corollary 1 can be found in Keilson [19] and Kijima [22], where the authors have used a similar approach as here. Also, the result can be found in Lemma 1 of Guillemin and Pinchon [13]. Note however that in the latter, the authors have proved their result through a totally different approach based on counting processes and the Strong Law of Large Numbers.

Corollary 2 Under Condition $C^{2}$, the variance $\operatorname{Var}\left(\theta_{m}\right)$ of the random variable $\theta_{m}$ is given, for $m \geq 1$, by

$$
\operatorname{Var}\left(\theta_{m}\right)=\frac{2}{\lambda_{m-1} \pi_{m-1}} \sum_{n=m+1}^{\infty} \frac{1}{\lambda_{n-1} \pi_{n-1}}\left(\sum_{i=n}^{\infty} \pi_{i}\right)^{2}+\frac{1}{\lambda_{m-1}^{2} \pi_{m-1}^{2}}\left(\sum_{i=m}^{\infty} \pi_{i}\right)^{2}
$$

Proof. The result here concerns the special case, $k=2$, of Theorem 1. For $k=1$, Equation (26) implies the following relation between the expected values of $\theta_{n}$ and $\theta_{n+1}$

$$
\bar{\theta}_{n}=\frac{1}{\mu_{n}}+\frac{\lambda_{n}}{\mu_{n}} \bar{\theta}_{n+1}, \quad n \geq 1
$$

Using Equations (19) and (32), we deduce that $V_{n, k}=2\left(\bar{\theta}_{n}\right)^{2}$, for $n \geq 1$ and $k=2$. Next, applying Corollary 1 , we obtain the second order moment $E\left(\theta_{m}^{2}\right)$ of the random variable $\theta_{m}$ as follows

$$
E\left(\theta_{m}^{2}\right)=\frac{2}{\lambda_{m-1} \pi_{m-1}} \sum_{n=m}^{\infty} \frac{1}{\lambda_{n-1} \pi_{n-1}}\left(\sum_{i=n}^{\infty} \pi_{i}\right)^{2}, \text { for } m \geq 1 .
$$

Finally, knowing that $\operatorname{Var}\left(\theta_{m}\right)=E\left(\theta_{m}^{2}\right)-\left(\bar{\theta}_{m}\right)^{2}$, the result holds immediately.

Note that the result in Corollary 2 can be found in Proposition 3.1 of Ball and Stefanov [6]. The authors have used a different approach by means of a random variable counting the number of distinct sojourns in system states.

Up to now, we computed the moments of the random variable $\theta_{m}$. In what follows, we go on to find explicit expressions for the moments of the random variable $\tau_{m}$. Recall that $\tau_{m}$ represents the upcrossing time from state $m-1$ to state $m$.

Theorem 2 The $k$ th order moment $E\left(\tau_{m}^{k}\right), k \geq 1$, of the random variable $\tau_{m}, m \geq 1$, is given by

$$
E\left(\tau_{m}^{k}\right)=\frac{1}{\lambda_{m-1} \pi_{m-1}} \sum_{n=1}^{m} \lambda_{n-1} \pi_{n-1} W_{n, k}
$$

where

$$
W_{n, k}=\left\{\begin{array}{l}
\frac{k !}{\lambda_{0}^{k}}, \text { for } n=1, k \geq 1, \\
\frac{k}{\lambda_{n-1}} E\left(\tau_{n}^{k-1}\right)+\frac{\mu_{n-1}}{\lambda_{n-1}} \sum_{j=1}^{k-1}\left(\begin{array}{c}
k \\
j
\end{array}\right) E\left(\tau_{n-1}^{j}\right) E\left(\tau_{n}^{k-j}\right), \text { for } n \geq 2, k \geq 1 .
\end{array}\right.
$$

Proof. See Appendix A. 
In Corollaries 3 and 4, we deduce the expectation and the variance of the random variable $\tau_{m}$, respectively.

Corollary 3 The expectation $\bar{\tau}_{m}$ of the random variable $\tau_{m}$ is given by

$$
\bar{\tau}_{m}=\frac{1}{\lambda_{m-1} \pi_{m-1}} \sum_{n=0}^{m-1} \pi_{n}, \text { for } m \geq 1
$$

Proof. For $n \geq 1$ and $k=1$, we have $W_{n, k}=\frac{1}{\lambda_{n-1}}$. Then, applying Theorem 2 completes the proof.

The result of Corollary 3 can be found in Keilson [19, 20], Sumita [32], and also in Lemma 1 of Guillemin and Pinchon [13].

Corollary 4 The variance $\operatorname{Var}\left(\tau_{m}\right)$ of the random variable $\tau_{m}$ is given by

$$
\operatorname{Var}\left(\tau_{m}\right)=\frac{2}{\lambda_{m-1} \pi_{m-1}} \sum_{n=1}^{m-1} \frac{1}{\lambda_{n-1} \pi_{n-1}}\left(\sum_{i=0}^{n-1} \pi_{i}\right)^{2}+\frac{1}{\lambda_{m-1}^{2} \pi_{m-1}^{2}}\left(\sum_{i=0}^{m-1} \pi_{i}\right)^{2}, \text { for } m \geq 1
$$

Proof. For $n \geq 1$ and $k=2$, we have $W_{n, k}=2\left(\bar{\tau}_{n}\right)^{2}$. Using Corollary 3 for the expression of $\bar{\tau}_{n}$, $n \geq 1$, and then applying Theorem 2 , we obtain the second order moment $E\left(\tau_{m}^{2}\right)$ of $\tau_{m}$ as follows

$$
E\left(\tau_{m}^{2}\right)=\frac{2}{\lambda_{m-1} \pi_{m-1}} \sum_{n=1}^{m} \frac{1}{\lambda_{n-1} \pi_{n-1}}\left(\sum_{i=0}^{n-1} \pi_{i}\right)^{2}, \text { for } m \geq 1 .
$$

Using $\operatorname{Var}\left(\tau_{m}\right)=E\left(\tau_{m}^{2}\right)-\left(\bar{\tau}_{m}\right)^{2}$ and Corollary 3 complete the proof.

In what follows, we use the results obtained above to derive the moments for the remaining transient characteristics defined in Section 2.1. Using the independence between the random variables $\theta_{i}$ and $\theta_{j}$ for $i, j \geq 1$, and Newton's binomial formula, we can obtain the closed-form expressions of the moments of $D_{m}$ and $U_{m}$. Recall that $D_{m}$ is the downcrossing time from state $m$ to the empty state 0 , and $U_{m}$ is the upcrossing time from state 0 to state $m$. For presentation issues, we only provide their expectations and variances. Note that the expressions of expectations can be found in Kijima [22], whereas the variance expressions we give below are to our knowledge new.

Under Condition $C^{1}$, let $\bar{D}_{m}\left(\bar{U}_{m}\right)$ be the expectation of the random variable $D_{m}\left(U_{m}\right)$, and under Condition $C^{2}$, let $\operatorname{Var}\left(D_{m}\right)\left(\operatorname{Var}\left(U_{m}\right)\right)$ be its variance. From Corollaries 1 and 3, we have respectively for $m \geq 1$,

$$
\begin{aligned}
& \bar{D}_{m}=\sum_{l=1}^{m} \frac{1}{\lambda_{l-1} \pi_{l-1}} \sum_{n=l}^{\infty} \pi_{n}, \\
& \bar{U}_{m}=\sum_{l=1}^{m} \frac{1}{\lambda_{l-1} \pi_{l-1}} \sum_{n=0}^{l-1} \pi_{n} .
\end{aligned}
$$

Now, using the independence, for any $i, j \geq 1$, between the random variables $\theta_{i}$ and $\theta_{j}$ on the one hand, and $\tau_{i}$ and $\tau_{j}$ on the other hand, we deduce respectively that $\operatorname{Var}\left(D_{m}\right)=\sum_{l=1}^{m} \operatorname{Var}\left(\theta_{l}\right)$, and 
$\operatorname{Var}\left(U_{m}\right)=\sum_{l=1}^{m} \operatorname{Var}\left(\tau_{l}\right)$. So, from Corollaries 2 and 4, we state respectively that, for $m \geq 1$,

$$
\begin{gathered}
\operatorname{Var}\left(D_{m}\right)=\sum_{l=1}^{m}\left(\frac{2}{\lambda_{l-1} \pi_{l-1}} \sum_{n=l+1}^{\infty} \frac{1}{\lambda_{n-1} \pi_{n-1}}\left(\sum_{i=n}^{\infty} \pi_{i}\right)^{2}+\frac{1}{\lambda_{l-1}^{2} \pi_{l-1}^{2}}\left(\sum_{i=l}^{\infty} \pi_{i}\right)^{2}\right) \\
\operatorname{Var}\left(U_{m}\right)=\sum_{l=1}^{m}\left(\frac{2}{\lambda_{l-1} \pi_{l-1}} \sum_{n=1}^{l-1} \frac{1}{\lambda_{n-1} \pi_{n-1}}\left(\sum_{i=0}^{n-1} \pi_{i}\right)^{2}+\frac{1}{\lambda_{l-1}^{2} \pi_{l-1}^{2}}\left(\sum_{i=0}^{l-1} \pi_{i}\right)^{2}\right)
\end{gathered}
$$

Consider now the random variable $C_{m}$ denoting the time between two visits by the process at state 0 , given that the process hits state $m$. With some algebra, the expectation $\bar{C}_{m}$ under Condition $C^{1}$ and the variance $\operatorname{Var}\left(C_{m}\right)$ under Condition $C^{2}$ of $C_{m}$, for $m \geq 1$, are given by

$$
\begin{gathered}
\bar{C}_{m}=\left(\sum_{n=0}^{\infty} \pi_{n}\right) \cdot\left(\sum_{l=1}^{m} \frac{1}{\lambda_{l-1} \pi_{l-1}}\right) \\
\operatorname{Var}\left(C_{m}\right)=\left(\sum_{l=1}^{m} \frac{2}{\lambda_{l-1} \pi_{l-1}}\right) \cdot\left(\sum_{n=1}^{\infty} \frac{1}{\lambda_{n-1} \pi_{n-1}}\left(\left(\sum_{i=0}^{n-1} \pi_{i}\right)^{2}+\left(\sum_{i=n}^{\infty} \pi_{i}\right)^{2}\right)\right) \\
+\sum_{l=1}^{m} \frac{1}{\lambda_{l-1}^{2} \pi_{l-1}^{2}}\left(\left(\sum_{i=0}^{l-1} \pi_{i}\right)^{2}+\left(\sum_{i=l}^{\infty} \pi_{i}\right)^{2}\right) .
\end{gathered}
$$

Let us come back to investigate the condition under which a birth-death process has ergodic degree $k, k \geq 1$. The quantities $D_{m}, m \geq 1$, play a key role to derive explicitly the Conditions $C^{k}$ for higher order moments, $k \geq 3$, which to the best of our knowledge do not exist in the literature. Let the random variable $D_{e, j}$ be the first passage time from the ergodic distribution to state $j$, $j \geq 0$. In accordance with the notations in Section 2.1, the random variable $D_{e}$ denotes the first passage time from the ergodic distribution to state 0 . It is clear that the $k$ th order moment of $D_{e}$, for $k \geq 1$, is given by

$$
E\left(D_{e}^{k}\right)=\sum_{s=0}^{\infty} p_{s} E\left(D_{s}^{k}\right)
$$

Recall that the quantities $\left\{p_{s}, s \geq 0\right\}$ are the stationary probabilities already given in Expression (3). Collecting thereafter some developments in Coolen-Schrijner and van Doorn [9], we state the following theorem.

Theorem 3 Condition $C^{k}, k \geq 1$, holds if and only if

$$
\sum_{s=0}^{\infty} p_{s} E\left(D_{s}^{k-1}\right)<\infty
$$

Proof. From Theorem 3.1 in Coolen-Schrijner and van Doorn [9], we state on the one hand that the $k$ th order moment of the first passage time from any state $i$ to any state $j$ is finite, if and only if, the $(k-1)$ th order moment of the first passage time from the ergodic distribution to any state $j$ is finite. On the other hand, the latter condition is sufficient and necessary for the $(k-1)$ th order moment 
of the first passage time from the ergodic distribution to some state $j$ to be finite. Applying this statement to the particular case, $j=0$, and using Equation (45) complete the proof.

As application, we give in Corollary 5 an explicit expression for Condition $C^{3}$.

Corollary 5 Condition $C^{3}$ holds if and only if

$$
\begin{aligned}
\sum_{s=0}^{\infty} \sum_{l=1}^{s} \frac{2 \pi_{s}}{\lambda_{l-1} \pi_{l-1} \sum_{j=0}^{\infty} \pi_{j}} \sum_{n=l}^{\infty} \frac{1}{\lambda_{n-1} \pi_{n-1}}\left(\sum_{r=n}^{\infty} \pi_{r}\right)^{2} & \\
& \quad+\sum_{s=0}^{\infty} \sum_{i, j=1, j>i}^{s} \frac{2 \pi_{s}}{\lambda_{i-1} \lambda_{j-1} \pi_{i-1} \pi_{j-1} \sum_{j=0}^{\infty} \pi_{j}}\left(\sum_{n=i}^{\infty} \pi_{n}\right)\left(\sum_{n=j}^{\infty} \pi_{n}\right)<\infty .
\end{aligned}
$$

Proof. See Appendix B.

We close the analysis for ordinary first passage times and turn to that of conditional first passage times.

\section{Moments of Conditional First Passage Times}

In this section, we focus on calculating the $k$ th order moment, $k \geq 1$, of the conditional first passage times defined in Section 2.2, ${ }^{r} \theta_{m}$ and ${ }^{r} \tau_{m}$. The results we derive here has not been done before in the literature, except as we shall mention later, for a special case for ${ }^{r} \tau_{m}$. Note that no existence conditions are required for the computation of their moments.

Before giving the results for the conditional random variables, we need to introduce some notations. These preliminaries are specifically related to the notion of ruin probabilities. Consider again the birth-death process defined in Section 2 . Let ${ }^{r} \eta_{m}$ be the ruin probability that the particle, starting at $m$, reaches $m-1$ first before $r, 1 \leq m<r$. It is clear that the ruin probability ${ }^{r} \eta_{r-1}$ to reach $r-2$ starting at $r-1$, without visiting $r$, is given by $\frac{\mu_{r-1}}{\lambda_{r-1}+\mu_{r-1}}$. For a given $m, 1 \leq m<r-1$, we define the event ${ }^{r} A_{m}$ that the particle reaches first $m-1$ starting from $m$, without visiting $r$. Let us calculate now the probability that ${ }^{r} A_{m}$ occurs, namely ${ }^{r} \eta_{m}$. In state $m$, two events can occur: either the process goes down to $m-1$, say event ${ }^{r} B_{m}$, or the process goes up to $m+1$ which is the complementary event of ${ }^{r} B_{m}$, say ${ }^{r} B_{m}^{c}$. Hence, we can write

$$
\operatorname{Pr}\left({ }^{r} A_{m}\right)=\operatorname{Pr}\left({ }^{r} A_{m} \mid{ }^{r} B_{m}\right) \cdot \operatorname{Pr}\left({ }^{r} B_{m}\right)+\operatorname{Pr}\left({ }^{r} A_{m} \mid{ }^{r} B_{m}^{c}\right) \cdot \operatorname{Pr}\left({ }^{r} B_{m}^{c}\right) .
$$

The event ${ }^{r} A_{m} \mid{ }^{r} B_{m}$ is to reach $m-1$ starting from $m-1$ without visiting $r$, which obviously occurs with probability 1 since the process is already in state $m-1$. The event ${ }^{r} A_{m} \mid{ }^{r} B_{m}^{c}$ is to reach $m-1$ first before $r$, starting at $m+1$, which is equivalent to the following: starting at $m+1$, the process reaches $m$ without visiting $r$, then starting from $m$, it reaches $m-1$ without visiting $r$. So, $\operatorname{Pr}\left({ }^{r} A_{m} \mid{ }^{r} B_{m}^{c}\right)={ }^{r} \eta_{m+1}{ }^{r} \eta_{m}$. Furthermore, the event ${ }^{r} B_{m}$ occurs with probability $\frac{\mu_{m}}{\lambda_{m}+\mu_{m}}$, and the event ${ }^{r} B_{m}^{c}$ with probability $\frac{\lambda_{m}}{\lambda_{m}+\mu_{m}}$. These arguments lead to the following recursive relation

$$
{ }^{r} \eta_{m}=\frac{\mu_{m}}{\lambda_{m}+\mu_{m}}+\frac{\lambda_{m}}{\lambda_{m}+\mu_{m}}{ }^{r} \eta_{m+1}{ }^{r} \eta_{m}, \text { for } 1 \leq m<r-1,
$$


or equivalently

$$
{ }^{r} \eta_{m}=\frac{\mu_{m}}{\mu_{m}+\lambda_{m}\left(1-{ }^{r} \eta_{m+1}\right)}, \text { for } 1 \leq m<r-1,
$$

starting with ${ }^{r} \eta_{r-1}=\frac{\mu_{r-1}}{\lambda_{r-1}+\mu_{r-1}}$.

For $1 \leq m<r-1$, we define the quantities $\delta_{m}$ by

$$
\delta_{m}=\mu_{m}+\lambda_{m}\left(1-{ }^{r} \eta_{m+1}\right)
$$

and for $0 \leq m<r-1$, we introduce the quantities $\chi_{m}$ as follows

$$
\chi_{0}=1, \text { and } \chi_{m}=\frac{\left(\lambda_{0}{ }^{r} \eta_{1}\right)\left(\lambda_{1}{ }^{r} \eta_{2}\right) \ldots\left(\lambda_{m-1}{ }^{r} \eta_{m}\right)}{\delta_{1} \delta_{2} \ldots \delta_{m}}, \text { for } 1 \leq m<r-1
$$

Theorem 4 The $k$ th order moment $E\left({ }^{r} \theta_{m}^{k}\right), k \geq 1$, of the random variable ${ }^{r} \theta_{m}, 1 \leq m \leq r-1$, is given by

$$
E\left({ }^{r} \theta_{r-1}^{k}\right)=\frac{k !}{\left(\lambda_{r-1}+\mu_{r-1}\right)^{k}}
$$

and

$$
E\left({ }^{r} \theta_{m}^{k}\right)=\frac{1}{\lambda_{m-1}{ }^{r} \eta_{m} \chi_{m-1}} \sum_{n=m}^{r-1} \lambda_{n-1}{ }^{r} \eta_{n} \chi_{n-1}{ }^{r} V_{n, k}, \text { for } 1 \leq m<r-1,
$$

where

$$
{ }^{r} V_{n, k}=\frac{k}{\delta_{n}} E\left({ }^{r} \theta_{n}^{k-1}\right)+\frac{\lambda_{n}{ }^{r} \eta_{n+1}}{\delta_{n}} \sum_{j=1}^{k-1}\left(\begin{array}{c}
k \\
j
\end{array}\right) E\left({ }^{r} \theta_{n}^{j}\right) E\left({ }^{r} \theta_{n+1}^{k-j}\right), \text { for } 1 \leq m<r-1 .
$$

Proof. One can easily see that the random variable ${ }^{r} \theta_{r-1}$ is exponentially distributed with rate $\lambda_{r-1}+\mu_{r-1}$. Then, its $k$ th order moment is given by $E\left({ }^{r} \theta_{r-1}^{k}\right)=\frac{k !}{\left(\lambda_{r-1}+\mu_{r-1}\right)^{k}}, k \geq 1$. For $1 \leq m<r-1$, we can write using the Strong Markov Property

$$
\begin{cases}{ }^{r} \theta_{m} \doteq \varepsilon_{\lambda_{m}+\mu_{m}} & , \text { with probability } 1-{ }^{r} \omega_{m} \\ { }^{r} \theta_{m} \doteq \varepsilon_{\lambda_{m}+\mu_{m}}+{ }^{r} \theta_{m+1}+{ }^{r} \hat{\theta}_{m} & , \text { with probability }{ }^{r} \omega_{m}\end{cases}
$$

where $\varepsilon_{\lambda_{m}+\mu_{m}}$ is a random variable exponentially distributed with parameter $\lambda_{m}+\mu_{m}$. The random variables ${ }^{r} \theta_{m},{ }^{r} \theta_{m+1}$ and ${ }^{r} \hat{\theta}_{m}$ are independent. In addition, the random variables ${ }^{r} \theta_{m}$ and ${ }^{r} \hat{\theta}_{m}$ are identically distributed. The quantity ${ }^{r} \omega_{m}$ is the probability that the process goes up to state $m+1$ and subsequently comes back to $m$ without visiting $r,{ }^{r} \omega_{m}=\frac{\lambda_{m}}{\lambda_{m}+\mu_{m}}{ }^{r} \eta_{m+1}$.

Let ${ }^{r} \tilde{\theta}_{m}(s)$ be the Laplace transform of the random variable ${ }^{r} \theta_{m}$. Then, Equation (56) yields

$$
\left(\lambda_{m}+\mu_{m}+s\right)^{r} \tilde{\theta}_{m}(s)=\delta_{m}+\lambda_{m}{ }^{r} \eta_{m+1}{ }^{r} \tilde{\theta}_{m+1}(s)^{r} \tilde{\theta}_{m}(s), \text { for } 1 \leq m<r-1 .
$$

As in the proof of Theorem 1, using Leibnitz's differentiation formula, we obtain the following recursive relation, for $1 \leq m<r-1, k \geq 1$

$$
E\left({ }^{r} \theta_{m}^{k}\right)=\frac{\lambda_{m}{ }^{r} \eta_{m+1}}{\delta_{m}} E\left({ }^{r} \theta_{m+1}^{k}\right)+{ }^{r} V_{m, k}
$$


Finally, with straightforward manipulations we complete the proof.

It should be noted that Theorem 1 can be seen as a special case of Theorem $4(r=\infty)$. An intuitive explanation is related to the comparison between the potential coefficients $\pi_{m}$ and $\chi_{m}$. For the analysis of the random variable $\theta_{m}$, we consider all possible paths to reach $m-1$, starting at $m$. However for the analysis of the conditional random variable ${ }^{r} \theta_{m}$, we only consider a subset included in the first set of paths. For this subset, the paths do not contain state $r$. One may see that fact when looking on the expressions of $\pi_{m}$ and $\chi_{m}\left(\lambda_{i}\right.$ is substituted by $\lambda_{i}{ }^{r} \eta_{i+1}$, and $\mu_{i}$ is substituted by $\left.\mu_{i}+\lambda_{i}\left(1-{ }^{r} \eta_{i+1}\right)\right)$. Naturally, the expression of $\pi_{m}$ does not involve any condition related to whether visiting state $r$ or not. However, each birth rate $\lambda_{i}$ (in the expression of $\pi_{m}$ ) is multiplied by ${ }^{r} \eta_{i+1}$ (in the expression of $\chi_{m}$ ), which gives the rate at which the particle moves up to $i+1$ starting at $i$ and when it will come back to $i$, it will not visit state $r$ in between. On the one hand, this explains the change in the birth rates. On the other hand, an explanation for the change in the death rates would be as follows. Since the total rate at which a particle leaves state $i$ is $\lambda_{i}+\mu_{i}$, the death rate corresponding to state $i$ in the expression of $\chi_{m}$ must respect that property. Thus, a birth rate $\mu_{i}$ in the expression of $\pi_{m}$ becomes $\delta_{i}=\mu_{i}+\lambda_{i}\left(1-{ }^{r} \eta_{i+1}\right)$ in the expression of $\chi_{m}$.

Corollary 6 The expectation ${ }^{r} \bar{\theta}_{m}$ of the random variable ${ }^{r} \theta_{m}$ is given by

$$
{ }^{r} \bar{\theta}_{r-1}=\frac{1}{\lambda_{r-1}+\mu_{r-1}}, \text { and, }{ }^{r} \bar{\theta}_{m}=\frac{1}{\lambda_{m-1}{ }^{r} \eta_{m} \chi_{m-1}} \sum_{n=m}^{r-1} \chi_{n}, \text { for } 1 \leq m<r-1 .
$$

Proof. The first part of the corollary is immediately obtained from the special case, $k=1$, of Theorem 4. As for the second part, one has ${ }^{r} V_{n, 1}=\frac{1}{\delta_{n}}$ for $1 \leq n<r-1$, next observing that $\lambda_{n-1}{ }^{r} \eta_{n} \chi_{n-1}=\delta_{n} \chi_{n}$ and again applying Theorem 4 , for $k=1$, complete the proof.

Corollary 7 The variance $\operatorname{Var}\left({ }^{r} \theta_{m}\right)$ of the random variable ${ }^{r} \theta_{m}$ is given by

$$
\begin{gathered}
\operatorname{Var}\left({ }^{r} \theta_{r-1}\right)=\frac{1}{\left(\lambda_{r-1}+\mu_{r-1}\right)^{2}}, \text { and, for } 1 \leq m<r-1, \\
\operatorname{Var}\left({ }^{r} \theta_{m}\right)=\frac{2}{\lambda_{m-1}{ }^{r} \eta_{m} \chi_{m-1}} \sum_{n=m+1}^{r-1} \frac{1}{\lambda_{n-1}{ }^{r} \eta_{n} \chi_{n-1}}\left(\sum_{i=n}^{r-1} \chi_{i}\right)^{2}+\frac{1}{\lambda_{m-1}^{2} \eta_{m}^{2} \chi_{m-1}^{2}}\left(\sum_{i=m}^{r-1} \chi_{i}\right)^{2} .
\end{gathered}
$$

Proof. The first part of the corollary is a direct consequence (special case, $k=2$ ) of Theorem 4 . For the second part, it suffices to see that ${ }^{r} V_{n, 2}=2\left({ }^{r} \bar{\theta}_{n}\right)^{2}, 1 \leq m<r-1$. Next, by simply applying Corollary 6 and again Theorem 4 , for $k=2$, we obtain the second order moment $E\left({ }^{r} \theta_{m}^{2}\right)$ of ${ }^{r} \theta_{m}$ as follows.

$$
E\left({ }^{r} \theta_{m}^{2}\right)=\frac{2}{\lambda_{m-1}{ }^{r} \eta_{m} \chi_{m-1}} \sum_{n=m}^{r-1} \frac{1}{\lambda_{n-1}{ }^{r} \eta_{n} \chi_{n-1}}\left(\sum_{i=n}^{r-1} \chi_{i}\right)^{2}, \text { for } 1 \leq m<r-1 .
$$

Finally, the result holds using the definition, $\operatorname{Var}\left({ }^{r} \theta_{m}\right)=E\left({ }^{r} \theta_{m}^{2}\right)-\left({ }^{r} \bar{\theta}_{m}\right)^{2}$. 
In what follows, we focus on the moments computation of the random variable ${ }^{r} \tau_{m}$. As above, we first introduce some notations. Let ${ }^{r} \nu_{m}$ be the ruin probability that the process, starting at $m-1$, reaches $m$ first before $r, m \geq r+2$. It is clear that ${ }^{r} \nu_{r+2}=\frac{\lambda_{r+1}}{\lambda_{r+1}+\mu_{r+1}}$. With a similar explanation as for the ruin probability ${ }^{r} \eta_{m}$, we give the following recursive relation, for $m>r+2$,

$$
{ }^{r} \nu_{m}=\frac{\lambda_{m-1}}{\lambda_{m-1}+\mu_{m-1}\left(1-{ }^{r} \nu_{m-1}\right)} .
$$

For $m \geq r+1$, we define the quantities $\beta_{m}$ by

$$
\beta_{r+1}=\lambda_{r+1}+\mu_{r+1} \text {, and, } \beta_{m}=\lambda_{m}+\mu_{m}\left(1-{ }^{r} \nu_{m}\right), \text { for } m>r+1,
$$

and for $m \geq r+1$, we introduce the quantities $\phi_{m}$ as

$$
\phi_{r+1}=1 \text {, and } \phi_{m}=\frac{\beta_{r+1} \beta_{r+2} \ldots \beta_{m-1}}{\left(\mu_{r+2}{ }^{r} \nu_{r+2}\right)\left(\mu_{r+3}{ }^{r} \nu_{r+3}\right) \ldots\left(\mu_{m}{ }^{r} \nu_{m}\right)} \text {, for } m>r+1 \text {. }
$$

Theorem 5 The $k$ th order moment $E\left({ }^{r} \tau_{m}^{k}\right), k \geq 1$, of the random variable ${ }^{r} \tau_{m}, m \geq r+2$, is given by

$$
E\left({ }^{r} \tau_{r+2}^{k}\right)=\frac{k !}{\left(\lambda_{r+1}+\mu_{r+1}\right)^{k}}
$$

and

$$
E\left({ }^{r} \tau_{m}^{k}\right)=\frac{1}{\beta_{m-1} \phi_{m-1}} \sum_{n=r+2}^{m} \beta_{n-1} \phi_{n-1}{ }^{r} W_{n, k}, \text { for } m>r+2
$$

where

$$
W_{n, k}=\frac{k}{\beta_{n-1}} E\left({ }^{r} \tau_{n}^{k-1}\right)+\frac{\mu_{n-1}{ }^{r} \nu_{n-1}}{\beta_{n-1}} \sum_{j=1}^{k-1}\left(\begin{array}{l}
k \\
j
\end{array}\right) E\left({ }^{r} \tau_{n-1}^{j}\right) E\left({ }^{r} \tau_{n}^{k-j}\right), \text { for } n>r+2, k \geq 1 .
$$

Proof. See Appendix C.

In Corollaries 8 and 9, we deduce the expectation and the variance of the random variable ${ }^{r} \tau_{m}$, respectively.

Corollary 8 The expectation ${ }^{r} \bar{\tau}_{m}$ of the random variable ${ }^{r} \tau_{m}$ is given by

$$
r_{\bar{\tau}_{m}}=\frac{1}{\beta_{m-1} \phi_{m-1}} \sum_{n=r+1}^{m-1} \phi_{n}, \text { for } m \geq r+2 \text {. }
$$

Proof. Observing that ${ }^{r} W_{n, 1}=\frac{1}{\beta_{n-1}}$, for $n \geq r+2$, the result holds from the special case, $k=1$, of Theorem 5 .

Corollary 9 The variance $\operatorname{Var}\left({ }^{r} \tau_{m}\right)$ of the random variable ${ }^{r} \tau_{m}$ is given, for $m \geq r+2$, by

$$
\operatorname{Var}\left({ }^{r} \tau_{m}\right)=\frac{2}{\beta_{m-1} \phi_{m-1}} \sum_{n=r+2}^{m-1} \frac{1}{\beta_{n-1} \phi_{n-1}}\left(\sum_{i=r+1}^{n-1} \phi_{i}\right)^{2}+\frac{1}{\beta_{m-1}^{2} \phi_{m-1}^{2}}\left(\sum_{i=r+1}^{m-1} \phi_{i}\right)^{2} .
$$


Proof. For $n \geq r+2$ and $k=2$, we have ${ }^{r} W_{n, k}=2\left({ }^{r} \bar{\tau}_{n}\right)^{2}$. Next, using Corollary 8 and applying Theorem 5 for the special case, $k=2$, give us the second order moment $E\left({ }^{r} \tau_{m}^{2}\right)$ of ${ }^{r} \tau_{m}$ as follows.

$$
E\left({ }^{r} \tau_{m}^{2}\right)=\frac{2}{\beta_{m-1} \phi_{m-1}} \sum_{n=r+2}^{m} \frac{1}{\beta_{n-1} \phi_{n-1}}\left(\sum_{i=r+1}^{n-1} \phi_{i}\right)^{2}, \text { for } m \geq r+2,
$$

which leads to the desired result.

Applying the above analysis, we again mention that we can obtain characteristics of several further random variables of conditional first passage times, such as conditional downcrossing and upcrossing times between two arbitrary states. These random variables are indeed easily expressed as a function of the random variables we analyze here, ${ }^{r} \theta_{m}$ and ${ }^{r} \tau_{m}$.

\section{Applications}

In this section, we give some applications of the results obtained in this paper. First, we revisit in Sections 5.1 and 5.2 the important concepts of busy period and busy cycle in queueing systems. Second, we address in Section 5.3 another important application, which is the prediction of statedependent queueing delays in non-standard queueing systems; systems with impatient customers (linear growth death rates), or state-dependent arrival rates, or in general, systems with statedependent transition rates.

In the applications below, we show how one may apply the results of this paper in order to derive the moments of various important practical random variables. We should note that computing the moments values are helpful for the characterization of the exact probability distribution of these random variables. Having only the first and second moments is important but often not sufficiently accurate to approximate an exact distribution. For instance, the third moment of a random variable allows to compute its skewness, and the fourth moment in turn allows to compute its kurtosis. The skewness is a measure of the "asymmetry" of a probability distribution, and the kurtosis is a measure of its "peakedness". For further details about these notions, we refer the reader to Joanes and Gill [14].

In general, such an analysis is related to the well known Moment Problem. The Moment Problem is the problem of finding a distribution whose moments have specified values, or of determining whether such a distribution exists. This area was first started by the works of Chebyshev through the well known Chebyshev inequalities. For a literature and historical perspective, see Bertsimas and Popescu [7]. For a more detailed discussion, the reader is referred to Akhiezer [3], and Shohat and Tamarkin [31].

\subsection{Busy Period in the $M / M / 1$ and $M / M / s$ Queues}

In this section, we apply some special cases of the results of Section 3 to retrieve known results for the simple $M / M / 1$ and $M / M / s$ queues.

First, let us consider an $M / M / 1$ queue. The arrival and service rates are $\lambda$ and $\mu$, respectively. We do not need here to specify the discipline of service under which the queue is working. Let $E(t)$ be the number of customers in system at a random instant $t$. The process $\{E(t), t \geq 0\}$ is 
a particular case of the birth-death process we present in Section 2. The birth rates are $\lambda_{m}=\lambda$, for $m \geq 0$, and the death rates are $\mu_{m}=\mu$, for $m \geq 1$. Let $\rho$ be the server utilization, $\rho=\lambda / \mu$. The conditions for our system to be ergodic and therefore to have an equilibrium solution for the stationary probabilities (Condition $C^{1}$ given in Relation (13)) are that

$$
\sum_{m=0}^{\infty}\left(\frac{\lambda}{\mu}\right)^{m}<\infty, \text { and } \sum_{m=0}^{\infty} \frac{1}{\lambda}\left(\frac{\mu}{\lambda}\right)^{m}=\infty
$$

The series on the left hand side of the first condition in Relation (72) will converge if and only if $\rho<1$. The second condition in Relation (72) will be satisfied if $\rho \leq 1$. Thus, the necessary and sufficient condition for ergodicity of degree 1 (Condition $C^{1}$ ) is simply $\rho<1$. Assume now that $\rho<1$ and let us consider the busy period duration of the $M / M / 1$ queue, namely the random variable $\theta_{1}$ as defined in Section 2.1. In the following, we check the results obtained in Section 3 for the particular system we present here.

On the one hand, we use the expressions found in Section 3 to compute the first three order moments of the random variable $\theta_{1}$. For our model, transition rates above one state do not depend on the state itself. Then, one should simplify the algebra using the fact that the random variables $\theta_{i}$ and $\theta_{j}$ are identically distributed, for $i, j \geq 1$. Next, by simply observing that $\sum_{i=1}^{\infty}\left(\frac{\lambda}{\mu}\right)^{i}=\frac{\lambda}{\mu-\lambda}$ (for $\frac{\lambda}{\mu}<1$ ), we deduce from Theorem 1 that for example the first three order moments are $\frac{1}{\mu-\lambda}$, $\frac{2 \mu}{(\mu-\lambda)^{3}}$ and $\frac{6 \mu(\lambda+\mu)}{(\mu-\lambda)^{5}}$, respectively. Note that Conditions $C^{2}$ and $C^{3}$ are identical to Condition $C^{1}$. From Relations (14) and (47), one may see that they hold if and only if $\rho<1$. On the other hand, it is known from classical results, as in Gross and Harris [11], that the Laplace transform in $t, \tilde{\theta_{1}}(s)$, of the probability density function (pdf) of the busy period duration of the $M / M / 1$ queue is given by $\tilde{\theta}_{1}(s)=\frac{2 \mu}{\lambda+\mu+s+\sqrt{(\lambda+\mu+s)^{2}-4 \lambda \mu}}$, for $s \geq 0$. Then, using the relation $E\left(\theta_{1}^{k}\right)=(-1)^{k} \tilde{\theta}_{1}^{(k)}(0)$, for $k \geq 1$, one can again find the expressions derived from our results.

The busy period results are of value when addressing the busy cycle analysis for the $M / M / 1$ queue. A busy cycle is defined as the sum of a busy period and an adjacent idle period, or equivalently, the time between two successive departures leaving an empty system, or two successive arrivals to an empty system. Since the arrivals here are assumed to follow a Poisson process, the probability density function (pdf) of the idle period is exponential with parameter $\lambda$; hence the pdf of the busy cycle for the $M / M / 1$ queue is the convolution of this negative exponential with the pdf of the busy period itself. Following the notations in Section 2.1, the busy cycle duration is clearly $\tau_{1}+\theta_{1}$, namely $C_{1}$. In particular, we deduce from Equation (43) that the busy cycle expectation is $\bar{C}_{1}=\frac{\mu}{\lambda(\mu-\lambda)}$, which agrees with a classical result in queueing literature, see for example Gross and Harris [11].

Let us now address the previous analysis for an $M / M / s$ queue. We consider an $M / M / s$ queue with $s$ identical and independent servers. We consider the same assumptions for the arrival and service processes as those for the $M / M / 1$ queue. Again, we do not need to specify the service discipline, except to be non-idling. Finally, let $\rho$ be the server utilization, $\rho=\lambda / s \mu$. Under stability condition, $C^{1}$, we have $\rho<1$.

The process $\{E(t), t \geq 0\}$ counting the number of customers in system is a birth-death process, 
and it is a particular case of the one we present in Section 2. The birth rates are $\lambda_{m}=\lambda$ for $m \geq 0$, and the death rates are $\mu_{m}=m \mu$ for $1 \leq m \leq s$, and $\mu_{m}=s \mu$ for $m>s$. The busy period of the $M / M / s$ system is defined as the time from an arrival of a customer to a system with only one idle server until the first time one of the servers becomes idle. Thus, it represents the duration of an excursion by the process $\{E(t), t \geq 0\}$ above the level $s-1$, namely $\theta_{s}$. With a little thought it should be clear that the busy period pdf of the $M / M / s$ queue can be obtained by taking its expression in the case of an $M / M / 1$ queue and substituting $\mu$ (capacity of service in the $M / M / 1$ queue) with $s \mu$ (capacity of service in the $M / M / s$ queue). One can easily validate this intuitive result by considering the state-transition-rate diagrams for both processes. In fact, transition rates, above any state $m \geq s-1$, of the birth-death process associated with the $M / M / s$ queue are constant. In addition, they reduce to the ones for the $M / M / 1$ case if we substitute $s \mu$ by $\mu$. In this configuration, both of the processes behave equivalently when calculating an excursion duration from state $m$ to state $m-1$, such that $m \geq s$, and in particular when calculating the busy period duration. Next, one may again check with some algebra that the expressions of the moments obtained from the results of Section 3 coincide with those already known from the literature.

\subsection{Busy Period in the $M / M / 1+M$ and $M / M / s+M$ Queues}

In this section, we address the analysis of the busy period for some special cases of queueing systems with reneging. Incorporating reneging in queueing models is well known to be of interest. It has an important effect on the performance measures. For instance, reneging is of special interest in manufacturing systems dealing with perishable products, in call centers where customers may hang up once they feel that their waiting time before getting service is too long, etc.

First, let us consider an $M / M / 1+M$ queue. The model is identical to the $M / M / 1$ queue described in Section 5.1. However, the customers here are impatient (symbol $M$ after the + ). After entering the queue, a customer will wait a random length of time for service to begin. If service has not begun by this time he will renege (leave the queue). Times before reneging are assumed to be mutually independent and identically exponentially distributed with rate $\sigma>0$. Again, we do not need for our analysis to specify the service discipline, except for it to be non-idling. For our $M / M / 1+M$ model, the corresponding infinitesimal transition rates in the generalized birth-death process are given by $\lambda_{m}=\lambda$ for $m \geq 0$, and $\mu_{m}=\mu+(m-1) \sigma$ for $m \geq 1$. Finally, we notice that abandonments make the system unconditionally stable, see for example Ward and Glynn [35]. In concrete terms, Condition $C^{1}$ is clearly satisfied for any set of parameters such that $\sigma>0$. One may also state this by observing that for any $\sigma>0$, there exists an integer $m_{0}$ such that for all $m \geq m_{0}$ we have $\lambda_{m} / \mu_{m}<1$. The integer $m_{0}$ is simply the smallest integer greater or equal to $\frac{\lambda-\mu}{\sigma}+1$.

The busy period duration is given by the random variable $\theta_{1}$. To obtain any moment of order $k$, for $k \geq 1$, it suffices to use the relations obtained in Theorem 1 . In what follows, we only give the expectation $\bar{\theta}_{1}$ and variance $\operatorname{Var}\left(\theta_{1}\right)$. From Corollary 1 , one state that

$$
\bar{\theta}_{1}=\frac{1}{\lambda} \sum_{n=1}^{\infty} \frac{\lambda^{n}}{\prod_{j=0}^{n-1}(\mu+j \sigma)} .
$$


Consider the Gamma Function $\Gamma(x)$ defined for $x \geq 0, \Gamma(x)=\int_{0}^{\infty} t^{x-1} e^{-t} d t$. It is known that $\prod_{j=0}^{n-1}(\mu+j \sigma)=\frac{\sigma^{n} \Gamma(\mu / \sigma+n)}{\Gamma(\mu / \sigma)}$, see Ancker and Gafarian [4]. So, from the relation $\gamma(a, x)=$ $e^{-a} a^{x} \sum_{n=0}^{\infty}\left(\frac{\Gamma(x)}{\Gamma(x+n+1)} a^{n}\right)$ where $\gamma(a, x)=\int_{0}^{a} t^{x-1} e^{-t} d t$ is the Incomplete Gamma Function defined for $a, x \geq 0$, we deduce with some algebra that

$$
\bar{\theta}_{1}=\frac{1}{\lambda} \cdot\left(\frac{\lambda}{\sigma}\right)^{1-\frac{\mu}{\sigma}} \cdot e^{\frac{\lambda}{\sigma}} \cdot \gamma\left(\frac{\lambda}{\sigma}, \frac{\mu}{\sigma}\right) .
$$

Note that Equation (74) can be useful for numerical computation since the Incomplete Gamma Function is extensively tabulated. The variance $\operatorname{Var}\left(\theta_{1}\right)$ is given by

$$
\operatorname{Var}\left(\theta_{1}\right)=\left(\bar{\theta}_{1}\right)^{2}+\frac{2}{\lambda^{2}} \sum_{n=2}^{\infty}\left(\frac{\prod_{j=0}^{n-2}(\mu+j \sigma)}{\lambda^{n-1}}\left(\sum_{i=n}^{\infty} \frac{\lambda^{i}}{\prod_{j=0}^{i-1}(\mu+j \sigma)}\right)^{2}\right)
$$

To get some numerical illustrations, we consider 3 cases with different system parameters. The parameters of the first $M / M / 1+M$ system are $\lambda=0.2, \mu=0.3$ and $\sigma=0.2$. Those for the second system are $\lambda=0.3, \mu=0.5$ and $\sigma=0.2$. Finally, we have for the third system $\lambda=0.8, \mu=0.5$ and $\sigma=0.4$. The results are shown in Table 1 .

\begin{tabular}{|c|c|c|c|c|c|c|}
\hline & \multicolumn{2}{|c|}{ System 1 } & \multicolumn{2}{c|}{ System 2 } & \multicolumn{2}{c|}{ System 3 } \\
\hline$k$ & Numer & Simu & Numer & Simu & Numer & Simu \\
\hline 1 & 5.15 & 5.15 & 3.24 & 3.24 & 5.66 & 5.66 \\
2 & 65.03 & 65.04 & 27.63 & 27.63 & 88.05 & 88.04 \\
3 & $1,319.64$ & $1,319.51$ & 392.12 & 392.14 & $2,157.46$ & $2,157.20$ \\
\hline
\end{tabular}

Table 1: $k$ th order moments of the busy period duration for the $M / M / 1+M$ queue, $k=1 . .3$

As one would expect, the busy period duration for the special case without abandonments $(\sigma=0)$ gives an upper bound of that we consider here. The reason is that reneging leads to fewer customers in the system. In Table 2, we give numerical examples for the first three order moments associated with the first two systems we consider above but without abandonments, $\sigma=0$. We omit the computation for the third system because it becomes unstable when assuming no abandonments.

\begin{tabular}{|c|c|c|}
\hline & \multicolumn{2}{|c|}{$E\left(\theta_{1}^{k}\right)$} \\
\hline$k$ & System 1 & System 2 \\
\hline 1 & 10 & 3.33 \\
2 & 600 & 37.03 \\
3 & 90,000 & 864.19 \\
\hline
\end{tabular}

Table 2: $k$ th order moments of the busy period duration for the $M / M / 1$ queue without abandonments, $k=1 . .3$

We notice that the analysis above can be easily extended to the case when the reneging rate depends on the position of the customer waiting in queue. Also, as we have explained for the $M / M / s$ queue, the busy period moments for the $M / M / s+M$ queue can be obtained simply by taking those of the $M / M / 1+M$ queue and substituting the service capacity, $\mu$, in the first model by that, $s \mu$, in the second model. 


\subsection{Estimating State-Dependent Delays}

In this section we present an application for computing state-dependent queueing delays in a multiclass Markovian queueing system. The motivation of this application is predicting queueing delays. Many prediction methods exist, see for example Whitt [36], Jouini et al. [15], Rosenlund [30], and Koole [24] where the author has developed an algorithm for calculating tail probabilities of Cox distributions.

Let us consider a multiclass multiserver queueing system. The system consists of $s$ identical and independent servers and two classes of impatient customers type $A$ and type $B$, each one with its own infinite queue. Interarrivals times are i.i.d and exponentially distributed with rates $\lambda_{A}$ (for type $A$ customers) and $\lambda_{B}$ (for type $B$ customers). For both types of customers, service times and times before reneging are i.i.d and exponentially distributed with rates $\mu$ and $\sigma$, respectively. Customers $A$ have a non-preemptive priority over customers $B$. Within each queue, the service discipline is First Come, First Served (FCFS). In what follows, we investigate predicting the state-dependent virtual delay of a newly arriving customer. The virtual delay is the time it takes for a new arrival to enter service (assuming he does not renege in between). We consider a new arrival who finds all servers busy, $n_{A}$ type $A$ waiting customers in queue $A$ and $n_{B}$ type $B$ customers in queue $B$. We separate the study depending on whether the call of interest is of type $A$ or $B$. Type $A$ customers observe a regular queue without priority. Then, the conditional waiting time distribution of a new customer $A$ is easy to derive. It follows an hypoexponential distribution, which is the convolution of $\left(n_{A}+1\right)$ exponential distributions with parameters $s \mu, s \mu+\sigma, s \mu+2 \sigma, \ldots$, and $s \mu+n_{A} \sigma$.

As for the conditional waiting time for a new type $B$ arrival, the analysis is more complicated because it is affected by future type $A$ arrivals. In the following, we revisit Section 3 to address that issue. Consider a new type $B$ arrival, and let $n_{T}$ be the total number of customers in the queues, $n_{T}=n_{A}+n_{B}$. We denote by $X_{n_{T}}^{B}$ the random variable representing his state-dependent virtual delay in queue. The latter is the time it takes for a server to become free for the customer of interest. In other words, it is the time until the $n_{A}+n_{B}$ waiting customers leave the queue (either start service or abandon the queue), plus the time for future type $A$ arrivals to either start service or abandon the queue, plus the duration for a server to become idle (when all servers are busy). Note that $X_{n_{T}}^{B}$ only do depend on $n_{T}$. The reason is that the rate of future type $A$ arrivals $\left(\lambda_{A}\right)$ does not depend on the number of type $A$ waiting customers in queue. Furthermore, the discipline of service is workconserving, and type $A$ and $B$ are statistically identical with respect to the memoryless service times and times before reneging. Hence, varying the quantities $n_{A}$ and $n_{B}$ so as $n_{A}+n_{B}$ is held constant, does not affect the waiting time distribution of the customer of interest.

To characterize $X_{n_{T}}^{B}$, one may formulate the problem as to calculate the downcrossing time until the first passage at state 0 , starting from state $n_{T}+1$, in a birth-death process with a constant birth rate, $\lambda_{m}=\lambda_{A}$ for $m \geq 0$, which represents future type $A$ arrivals during the waiting of the customer of interest, and with a death rate $\mu_{m}=s \mu+(m-1) \sigma$ for $m \geq 1$. We do not consider future type $B$ arrivals because the discipline of service within queue $B$ is FCFS. Thereafter using Equations (39) and (41), we give the expressions of the mean, $E\left(X_{n_{T}}^{B}\right)$, and the variance, $\operatorname{Var}\left(X_{n_{T}}^{B}\right)$, 
of $X_{n_{T}}^{B}$ as follows

$$
\begin{gathered}
E\left(X_{n_{T}}^{B}\right)=\frac{1}{\lambda_{A}}\left(\sum_{i=1}^{n_{T}+1} \frac{1}{\pi_{i-1}} \sum_{j=i}^{\infty} \pi_{j}\right), \\
\operatorname{Var}\left(X_{n_{T}}^{B}\right)=\frac{1}{\lambda_{A}^{2}}\left(2 \sum_{i=1}^{n_{T}+1} \frac{1}{\pi_{i-1}} \sum_{j=i+1}^{\infty} \frac{1}{\pi_{j-1}}\left(\sum_{l=j}^{\infty} \pi_{l}\right)^{2}+\sum_{i=1}^{n_{T}+1} \frac{1}{\pi_{i-1}^{2}}\left(\sum_{j=i}^{\infty} \pi_{j}\right)^{2}\right),
\end{gathered}
$$

where the quantities $\pi_{i}$ are defined as

$$
\pi_{0}=1, \text { and } \pi_{i}=\frac{\lambda_{A}^{i}}{\prod_{j=0}^{i-1}(s \mu+j \sigma)}, \text { for } i \geq 1
$$

Furthermore, the analysis can be extended to the case of more than 2 customer classes with non-preemptive strict priority. Consider the previous model but with $k$ customer classes, $k \geq 3$. Without loss of generality, we denote each class by the rank $i, 1 \leq i \leq k$, according to its priority level (the lower rank for the higher priority). To obtain the conditional waiting time characteristics for a new type $k$ arrival $(k \geq 3)$, we aggregate all the classes $i$ having the priority over the one of interest (all $i$ such that $1 \leq i<k$ ) into one class, next we pick up the same analysis as that for type $B$. The class aggregation is justified by the fact that the waiting time distribution of the customer of interest is not affected by the order of service of the customers having higher priority.

\section{Conclusions and Perspectives}

We focused on the transient behavior analysis of a general birth-death process. We gave closed-form expressions for the moments of important state-dependent characteristics. The characteristics deal with the random variables of ordinary and conditional first passage times. We derived several new expressions of the moments of the defined hitting and return times. Furthermore, we retrieved some known results as special cases. We also discussed the condition under which a birth-death process is said to be ergodic degree $k$. In particular, we gave a new explicit expression for the condition of ergodicity degree 3 . In the second part of the paper, we investigated possible applications of the results for some Markovian queueing models.

Several further applications could be also possible. For instance, deriving the stationary waiting time moments for some Markovian model where the arrival rate depend on the system state. Concretely, for example in a system where a new customer has a state-dependent probability to join the queue, which is the case for many systems in practice. To do so, we may compute the state-dependent waiting times as shown in this work. Thereafter, we derive the desired stationary $k$ th order moment of queueing delays, by averaging on all states seen by arrivals. It would be also interesting in practice to investigate approximations or numerical methods to avoid possible computation difficulties.

\section{Acknowledgements}

The authors would like to express their gratitude to two anonymous referees for their several useful suggestions to improve this paper, as well as Eric A. Van Doorn and Bruno Sericola for their helpful comments on an earlier version. 


\section{Appendix}

In this appendix, we present the proofs of Theorem 2, Corollary 5 and Theorem 5 .

\section{A Proof of Theorem 2}

From the Strong Markov Property, we can write, for $m \geq 2$

$$
\begin{cases}\tau_{m} \doteq \varepsilon_{\lambda_{m-1}+\mu_{m-1}} & \text {, with probability } \frac{\lambda_{m-1}}{\lambda_{m-1}+\mu_{m-1}} \\ \tau_{m} \doteq \varepsilon_{\lambda_{m-1}+\mu_{m-1}}+\tau_{m-1}+\hat{\tau}_{m} & \text {, with probability } \frac{\mu_{m-1}}{\lambda_{m-1}+\mu_{m-1}} .\end{cases}
$$

where $\varepsilon_{\lambda_{m-1}+\mu_{m-1}}$ is a random variable exponentially distributed with parameter $\lambda_{m-1}+\mu_{m-1}$. The random variables $\tau_{m}, \tau_{m-1}$ and $\hat{\tau}_{m}$ are independent. In addition, the random variables $\tau_{m}$ and $\hat{\tau}_{m}$ are identically distributed.

Let $\tilde{\tau}_{m}(s)$ be the Laplace transform of the random variable $\tau_{m}$. Then, Equation (79) yields

$$
\left(\lambda_{m}+\mu_{m}+s\right) \tilde{\tau}_{m+1}(s)=\lambda_{m}+\mu_{m} \tilde{\tau}_{m}(s) \tilde{\tau}_{m+1}(s), \text { for } m \geq 1
$$

Let $\tilde{\tau}_{m}^{(k)}(s)$ be the $k$ th derivative in $s$ of $\tilde{\tau}_{m}(s)$. Taking the $k$ th derivative in $s$ of both sides in Equation (80) using Leibnitz's differentiation formula, we obtain for $m \geq 1, k \geq 1$

$$
\left(\lambda_{m}+\mu_{m}+s\right) \tilde{\tau}_{m+1}^{(k)}(s)+k \tilde{\tau}_{m+1}^{(k-1)}(s)=\mu_{m} \sum_{j=0}^{k}\left(\begin{array}{l}
k \\
j
\end{array}\right) \tilde{\tau}_{m}^{(j)}(s) \tilde{\tau}_{m+1}^{(k-j)}(s) .
$$

For $m \geq 1$ and $j=0, \tilde{\tau}_{m}^{(j)}(0)=1$. For $m \geq 1$ and $j \geq 1, \tilde{\tau}_{m}^{(j)}(0)=(-1)^{j} E\left(\tau_{m}^{j}\right)$. Next, with some algebra, Equation (81) becomes for $s=0$

$$
E\left(\tau_{m+1}^{k}\right)=\frac{\mu_{m}}{\lambda_{m}} E\left(\tau_{m}^{k}\right)+W_{m+1, k}, \quad m \geq 1, \quad k \geq 1 .
$$

Since the random variable $\tau_{1}$ is exponentially distributed with rate $\lambda_{0}$, so $E\left(\tau_{1}^{k}\right)=\frac{k !}{\lambda_{0}^{k}}$. Then, $E\left(\tau_{1}^{k}\right)=W_{1, k}$, and backward manipulations in Relation (82) imply

$$
E\left(\tau_{m}^{k}\right)=\sum_{i=1}^{m}\left(\prod_{j=i}^{m-1} \frac{\mu_{j}}{\lambda_{j}}\right) W_{i, k}, \text { for } m \geq 1, k \geq 1 .
$$

Observing again that $\frac{1}{\mu_{n}} \prod_{j=n}^{m-1} \frac{\mu_{j}}{\lambda_{j}}=\frac{\pi_{n}}{\lambda_{m-1} \pi_{m-1}}$, for $m \geq n \geq 1$, we finally state that

$$
E\left(\tau_{m}^{k}\right)=\frac{1}{\lambda_{m-1} \pi_{m-1}} \sum_{n=1}^{m} \lambda_{n-1} \pi_{n-1} W_{n, k}, \text { for } m \geq 1, k \geq 1 .
$$

This completes the proof of the theorem. 


\section{B Proof of Corollary 5}

From Theorem 3, Condition $C^{3}$ holds if and only if

$$
\sum_{s=0}^{\infty} p_{s} E\left(D_{s}^{2}\right)<\infty
$$

where the stationary probabilities $p_{s}, s \geq 0$, are given in Expression (3).

From the independence between the random variables $\theta_{i}$ and $\theta_{j}$, for $i, j \geq 1$ and $i \neq j$, we have

$$
E\left(D_{s}^{2}\right)=\sum_{l=1}^{s} E\left(\theta_{l}^{2}\right)+2 \sum_{i, j=1, j>i}^{s} E\left(\theta_{i}\right) E\left(\theta_{j}\right)
$$

Using the above equation, Relation (85) becomes

$$
\sum_{s=0}^{\infty} \sum_{l=1}^{s} E\left(\theta_{l}^{2}\right)+\sum_{s=0}^{\infty} \sum_{i, j=1, j>i}^{s} 2 E\left(\theta_{i}\right) E\left(\theta_{j}\right)<\infty
$$

Substituting $E\left(\theta_{i}\right)$ by its expression in Corollary 1 and $E\left(\theta_{l}^{2}\right)$ by that given in Equation (33), we immediately get

$$
\begin{aligned}
\sum_{s=0}^{\infty} \sum_{l=1}^{s} \frac{2 \pi_{s}}{\lambda_{l-1} \pi_{l-1} \sum_{j=0}^{\infty} \pi_{j}} \sum_{n=l}^{\infty} \frac{1}{\lambda_{n-1} \pi_{n-1}}\left(\sum_{r=n}^{\infty} \pi_{r}\right)^{2} & \\
& \quad+\sum_{s=0}^{\infty} \sum_{i, j=1, j>i}^{s} \frac{2 \pi_{s}}{\lambda_{i-1} \lambda_{j-1} \pi_{i-1} \pi_{j-1} \sum_{j=0}^{\infty} \pi_{j}}\left(\sum_{n=i}^{\infty} \pi_{n}\right)\left(\sum_{n=j}^{\infty} \pi_{n}\right)<\infty .
\end{aligned}
$$

\section{Proof of Theorem 5}

It is easy to see that the $k$ th order moment of the random variable ${ }^{r} \tau_{r+2}$ is given by $E\left({ }^{r} \tau_{r+1}^{k}\right)=$ $\frac{k !}{\left(\lambda_{r+1}+\mu_{r+1}\right)^{k}}, k \geq 1$. For $m>r+2$, we can write from the Strong Markov Property

$$
\begin{cases}{ }^{r} \tau_{m} \doteq \varepsilon_{\lambda_{m-1}+\mu_{m-1}} & \text {, with probability } 1-{ }^{r} \alpha_{m-1} \\ { }^{r} \tau_{m} \doteq \varepsilon_{\lambda_{m-1}+\mu_{m-1}}+{ }^{r} \tau_{m-1}+{ }^{r} \hat{\tau}_{m} & , \text { with probability }{ }^{r} \alpha_{m-1}\end{cases}
$$

where $\varepsilon_{\lambda_{m-1}+\mu_{m-1}}$ is a random variable exponentially distributed with parameter $\lambda_{m-1}+\mu_{m-1}$. The random variables ${ }^{r} \tau_{m},{ }^{r} \tau_{m-1}$ and ${ }^{r} \hat{\tau}_{m}$ are independent. In addition, the random variables ${ }^{r} \tau_{m}$ and ${ }^{r} \hat{\tau}_{m}$ are identically distributed. The quantity ${ }^{r} \alpha_{m}$ is the probability that the process goes down from state $m$ to state $m-1$ and subsequently comes back to $m$ without visiting $r$, ${ }^{r} \alpha_{m}=\frac{\mu_{m}}{\lambda_{m}+\mu_{m}}{ }^{r} \nu_{m}$.

Using Laplace transforms and Leibnitz's differentiation formula, we obtain

$$
E\left({ }^{r} \tau_{m+1}^{k}\right)=\frac{\mu_{m}{ }^{r} \nu_{m}}{\beta_{m}} E\left({ }^{r} \tau_{m}^{k}\right)+{ }^{r} W_{m+1, k}, \quad m>r+2, \quad k \geq 1 .
$$

From the latter recursive relation, the result of the theorem follows.

Note that the general recursive relation, given in Equation (90), can be found in Sumita [32] in 
the special cases, $k=1$ and $k=2$.

\section{References}

[1] J. Abate and W. Whitt. Transient Behavior of the M/M/1 Queue via Laplace Transforms. Advances in Applied Probability, 20:145-178, 1988.

[2] J. Abate and W. Whitt. Calculating Time-Dependent Performance Measures for the M/M/1 Queue. IEEE Transactions on Communications, 37:1102-1104, 1989.

[3] N.I. Akhiezer. The Classical Moment Problem and Some Related Questions in Analysis. Hafner Publishing Co., New York, 1965. Translated by N. Kemmer.

[4] C. J. Ancker and A. Gafarian. Queueing with Impatient Customers Who Leave at Random. Journal of Industrial Engineering, 13:84-90, 1962.

[5] N. T. J. Bailey. A Continuous Time Treatment of a Single Queue Using Generating Functions. J. Roy. Statist. Soc. Ser., 16:288-291, 1954.

[6] F. Ball and V.T. Stefanov. Further Approaches to Computing Fondamental Characteristics of Birth-Death Processes. Journal of Applied Probability, 38:995-1005, 2001.

[7] D. Bertsimas and I. Popescu. Optimal Inequalities in Probability Theory: A Convex Optimization Approach. SIAM Journal on Optimization, 15:780-804, 2005.

[8] D. G. Champernowne. An Elementary Method of Solution of the Queueing Problem with a Single Server and a Constant Parameter. J. Roy. Statist. Soc. Ser., 18:125-128, 1956.

[9] P. Coolen-Schrijner and E.A. van Doorn. The Deviation Matrix of a Continuous-Time Markov Chain. Probability in the Engineering and Informational Sciences, 16:351-366, 2002.

[10] P. Flajolet and F. Guillemin. The Formal Teory of Birth-and-Death Processes, Lattice Path Combinatorics and Continued Fractions. Advances in Applied Probability, 32:750-778, 2000.

[11] D. Gross and C.M. Harris. Fundamentals of Queueing Theory. Wiley series in probability and mathematical statistics, 1985. 2rd Edition.

[12] F. Guillemin. Spectral Analysis of Birth and Death Processes. 2005. Working paper, submitted to Journal of Applied Probability.

[13] F. Guillemin and D. Pinchon. Excursions of Birth and Death Processes, Orthogonal Polynomials, and Continued Fractions. Journal of Applied Probability, 36:752-770, 1999.

[14] D.N. Joanes and C.A. Gill. Comparing Measures of Sample Skewness and Kurtosis. Journal of the Royal Statistical Society (Series D): The Statistician, 47:183-189, 1998.

[15] O. Jouini, Y. Dallery, and O. Z. Akşin. Modeling Call Centers with Delay Information. 2006. Working paper, Ecole Centrale Paris and Koç University.

[16] S. Karlin and J. McGregor. The Classification of Birth and Death Processes. Trans. Amer. Math. Soc., 86:366-401, 1957.

[17] S. Karlin and J. McGregor. The Differential Equation of Birth and Death Processes, and the Setieltjes Moment Problem. Trans. Amer. Math. Soc., 85:489-546, 1957.

[18] J. Keilson. A Review of Transient Behavior in Regular Diffusion and Birth-Death Processes. Part i. Journal of Applied Probability, 1:247-266, 1964. 
[19] J. Keilson. A Review of Transient Behavior in Regular Diffusion and Birth-Death Processes. Part ii. Journal of Applied Probability, 1:247-266, 1964.

[20] J. Keilson. Markov Chain Models - Rarity and Exponentiality. Springer-Verlag, New York, 1979.

[21] J. Keilson. On the Unimodality of Passage Time Densities in Birth-Death Processes. Statist. Neerlandica, 35:49-55, 1981.

[22] M. Kijima. Markov Processes for Stochastic Modeling. Chapman \& Hall, 1997. First Edition.

[23] L. Kleinrock. Queueing Systems, Theory, volume I. A Wiley-Interscience Publication, 1975.

[24] G. Koole. A Formula for Tail Probabilities of Cox Distributions. Journal of Applied Probability, 41:935-938, 2004.

[25] A. Krinik and Y. Sourouri. Taylor Series Solutions of Classical Queueing Systems. Abstracts American Mathematical Society, 11, 1990.

[26] P. Leguesdron, J. Pellaumail, G. Rubino, and B. Sericola. Transient Analysis of the M/M/1 Queue. Advances in Applied Probability, 25:702-713, 1993.

[27] M. Loève. Probability Theory I. Springer-Verlag, New York, 1977. 4rd Edition.

[28] Y. H. Mao. Ergodic Degrees For Continuous-Time Markov Chains. Science in China Ser. A, 47:161-174, 2004.

[29] P. R. Parthasarathy. A Transient Solution to a M/M/1 Queue: A New Simple Approach. Advances in Applied Probability, 19:997-998, 1987.

[30] S. I. Rosenlund. Upwards Passage Times in the Non-Negative Birth-Death Process. Scand. J. Statist., 4:90-92, 1977.

[31] J.A. Shohat and J.D. Tamarkin. The Problem of Moments. American Mathematical Society Mathematical surveys, Vol. II, American Mathematical Society, New York, 1943.

[32] U. Sumita. On Conditional Passage Time Structure of Birth-Death Processes. Journal of Applied Probability, 21:10-21, 1984.

[33] L. Takâcs. Introduction to the Theory of Queues. Oxford University Press, 1960.

[34] A. M. K. Tarabia. Transient Analysis of M/M/1/N Queue - An Alternative Approach. Tamkang Journal of Science and Engineering, 3:263-266, 2000.

[35] A.R. Ward and P.W. Glynn. A Diffusion Approximation for a Markovian Queue with Reneging. Queueing Systems: Theory and Applications (QUESTA), 43:103-128, 2003.

[36] W. Whitt. Predicting Queueing Delays. Management Science, 45:870-888, 1999. 